\title{
Fenoterol e salbutamol via inalatória em cães: aspectos clínico, hemogasométrico e eletrocardiográfico
}

\author{
[Fenoterol and salbutamol inhalation in dogs: clinical, haemogasometric and \\ electrocardiographic aspects] \\ R.M. Viel ${ }^{1}$, R.M.B. Nogueira ${ }^{2}$, V.M. Kaneko ${ }^{1}$, A. Melchert $^{2}$, C.B. Laposy ${ }^{2}$, \\ D.S. Clemente ${ }^{3}$, E. Franz ${ }^{2}$ \\ ${ }^{1}$ Aluno de pós-graduação - Universidade do Oeste Paulista - Presidente Prudente, SP \\ ${ }^{2}$ Universidade do Oeste Paulista - Presidente Prudente, SP \\ ${ }^{3}$ Aluno de graduação - Universidade do Oeste Paulista - Presidente Prudente, SP
}

\begin{abstract}
RESUMO
Avaliaram-se, por meio de exame clínico, hemogasométrico e eletrocardiográfico, os efeitos do salbutamol e do fenoterol, administrados por via inalatória em cães. Doze cães foram distribuídos em dois grupos: os do grupo FE receberam fenoterol na dose de 2 gotas $/ 5 \mathrm{~kg}$ de peso vivo, diluídas em solução de cloreto de sódio $0,9 \%$ por aparelho de inalação, e os do grupo SA receberam salbutamol pelo dosador de aerossol, na dose de $100 \mu \mathrm{g}$. Foram avaliados: frequência cardíaca (FC), frequência respiratória (FR), temperatura retal (TR), pressão arterial sistólica (PAS), hemogasometria e eletrocardiograma antes e após 30min, duas horas e seis horas do uso dos fármacos. Discreta estimulação cardíaca ocorreu nos animais do grupo SA duas horas após sua administração em relação ao momento-controle, e tremores foram predominantes nestes animais. Diminuição da PAS e aumento da FR foram observados nos dois grupos, e não houve alteração significativa da onda $\mathrm{T}$, da hemogasometria e do eletrocardiograma em ambos os grupos. O fenoterol provocou menor estimulação cardíaca e menos tremores comparado ao salbutamol, foi mais seguro e houve maior facilidade, menor custo e menor gasto de tempo na administração do salbutamol por inalador dosimetrado em relação ao fenoterol por nebulização.
\end{abstract}

Palavras-chave: cão, fenoterol, salbutamol, inalação

\begin{abstract}
Through physical examination, blood gas and the electrocardiographic effects of salbutamol and fenoterol, administered by inhalation in dogs was assessed. Twelve dogs were distributed into two groups: EF group animals received a fenoterol dose of 2 drops $/ 5 \mathrm{~kg}$ bodyweight, diluted in sodium chloride $0.9 \%$ inhalation device and animals in the SA group received salbutamol through aerosol feeder at a dose of $100 \mu \mathrm{g}$. Rectal temperature (RT), heart rate (HR), respiratory rate (RR), systolic blood pressure (SBP), blood gas and electrocardiogram before and after 30min., $2 h$ and $6 h$ after drug were evaluated. Mild cardiac stimulation occurred in SA group animals 2 hours after its administration compared to the control group, and tremors were predominant in these animals. A decrease in SBP and an increase in RR were observed in both animal groups and no significant alteration of the $T$ wave in the electrocardiogram and blood gas analysis in both groups were observed. The fenoterol caused less cardiac pacing and shivering compared to salbutamol, which was more secure. However, there was more ease, lower cost and less time spent in the administration of salbutamol administered through metereddose inhaler compared to fenoterol nebulization.
\end{abstract}

Keywords: dog, fenoterol, salbutamol, inhalation

Recebido em 16 de março de 2010

Aceito em 5 de setembro de 2011

E-mail: rosa@unoeste.br 


\section{INTRODUÇÃO}

Os agonistas $\beta_{2}$ são broncodilatadores de primeira escolha para o alívio dos sintomas das doenças respiratórias e têm-se mostrado eficazes e bem tolerados como tratamento de crises de broncoespasmo, doença pulmonar obstrutiva crônica e crises de asma (Zanoni e Palhares, 2002; Zangirolami Filho et al., 2008). Os agonistas $\beta_{2}$ ativam receptores específicos, que levam ao aumento na concentração intracelular de 3'5'adenosinamonofosfato cíclico (AMPc), os quais ativam canais de potássio por fosforilação. A abertura desses canais de potássio resulta em hiperpolarização celular e em inibição do influxo de cálcio, com consequente broncodilatação (Amantéa et al., 2002).

Salbutamol e fenoterol são agonistas $\beta_{2}$ seletivos de curta duração e parecem ter ação alfa e beta-1 muito reduzida, no entanto este efeito é dosedependente (Vieira et al., 2000; Gonzáles-Munoz et al., 2009). A aerossolterapia ou inalação de uma substância medicamentosa é um método de administração em que os fármacos são depositados diretamente no trato respiratório, permitindo a utilização de uma dosagem menor, além de ter rápida ação e diminuir a incidência dos efeitos colaterais de toxicidade sistêmica (Silva et al., 2001; Rota et al., 2007). Os inaladores diferenciam-se quanto ao grau de deposição pulmonar, mas sua eficácia depende também da técnica de inalação e do grau de obstrução das vias aéreas (Mendes-Campos e Biagioni, 2001). Frente à escassez de dados sobre o uso de $\beta_{2}$ agonistas em cães, os objetivos deste estudo foram avaliar e comparar os aspectos clínico, hemogasométrico e eletrocardiográfico dos broncodilatadores de curta ação, fenoterol e salbutamol, administrados por via inalatória.

\section{MATERIAL E MÉTODOS}

O estudo foi protocolado com o número 062/07 e aprovado pelo Comitê de Ética em Pesquisa da Instituição de origem.

Doze cães, de ambos os sexos, sem raça definida, adultos, com peso entre 5 e $12 \mathrm{~kg}$, cedidos pelo canil central da Instituição de origem, foram escolhidos por meio da normalidade do exame físico, hemograma, contagem de plaquetas e pesquisa de hematozoário, e distribuídos em dois grupos (FE e SA) de seis animais. Os do grupo FE receberam fenoterol (Bromidrato de fenoterol, SEM) por via inalatória na dose de 2 gotas $/ 5 \mathrm{~kg}$ de peso vivo, diluído em $5 \mathrm{~mL}$ de solução de cloreto de sódio $0,9 \%$ (solução isotônica de cloreto de sódio $0,9 \%$, Segmenta) e administrado por meio de nebulizador pneumático (Nebulizador pneumático, $\mathrm{NS}, \mathrm{St}_{3}$ ) durante 20 minutos. Os do grupo SA receberam salbutamol (Aerolin Apray, Glaxo Smith Kline) por meio de dosador de aerossol, na dose de $100 \mu \mathrm{g}$, acoplado a um espaçador. A técnica utilizada neste estudo para administração do salbutamol foi adaptada da técnica utilizada por Souza e Rossi (2005) em gatos.

Os períodos de observação ocorreram antes da administração dos fármacos - momento controle (MC) - e após 30 minutos (M30), duas horas (M2) e seis horas (M6) da administração dos fármacos. Foram avaliados: frequência cardíaca (FC), frequência respiratória (FR), temperatura retal (TR), pressão arterial sistólica (PAS) não invasiva utilizando aparelho Doppler ultrassônico com transdutor do tipo caneta (MEDPEJ, DV-2000), hemogasometria de sangue venoso processado em aparelho de gasometria (modelo Cobasb 121 Roche) e eletrocardiograma (modelo Cardiotest EK 51).

Os dados foram submetidos ao teste de Komogorov e Smirnov para comprovar normalidade. Para avaliar os diferentes momentos das varáveis não paramétricas variáveis classificadas por escores - e variáveis paramétricas, empregou-se análise de variância, e para comparação entre grupos, o test t (Pagano e Gauvreau, 2004). Todas as análises estatísticas foram realizadas empregando-se $o$ pacote computacional GraphPad InStat ${ }^{\circledR}$ versão 3.0. O nível de significância adotado para todas as comparações foi de $5 \%$.

\section{RESULTADOS E DISCUSSÃO}

As médias da frequência cardíaca dos grupos $\mathrm{FE}$ e SA mantiveram-se dentro da normalidade para a espécie em todos os momentos de avaliação (Tab. 1). Foi observado aumento da FC em M30 em um animal $(16,7 \%)$ do grupo FE e em dois animais $(33,3 \%)$ do grupo SA. Um dos animais do grupo SA permaneceu com a frequência elevada até M6, e o aumento da frequência 
cardíaca neste grupo foi significativo $(\mathrm{P}<0,05)$ em M2 em relação ao MC. O aumento da frequência cardíaca está relacionado à estimulação de receptores $\beta_{1}$ no coração, apesar da seletividade do grupo SA aos receptores $\beta_{2}$. Amantéa et al. (2002) relataram, em um trabalho de revisão, que alguns autores observaram estimulação cardíaca após nebulização com agonistas $\beta_{2}$.

Zanoni e Palhares (2002) obtiveram resultados semelhantes em crianças com o uso de agonistas $\beta 2$, momentos após o início da nebulização, efeito que permaneceu até 60 minutos. Apesar de a diferença entre os grupos não ter sido significativa $(\mathrm{P}>0,05)$ quanto à $\mathrm{FC}$, observou-se que os animais do grupo $\mathrm{FE}$ apresentaram menos estimulação cardíaca e efeito mais longo quando comparados aos do grupo SA, sugerindo maior segurança deste fármaco no que se refere a esse efeito colateral indesejável, diferente do observado por Tsoi e Apkhipov (2002), que relataram maior efeito sobre a frequência cardíaca do fenoterol em relação ao salbutamol.

Outros efeitos colaterais, como agitação, tremores, náusea e vômito, também foram relatados por outros autores em humanos (Zanoni e Palhares, 2002; Abramson et al., 2003). Neste estudo, foram observados tremores em quatro animais do grupo SA em M30, efeito que permaneceu até M6; no grupo FE, o efeito de tremor foi notado em um animal no $\mathrm{MC}$ e em três animais em M30 e M2, sendo que em um deles o tremor manteve-se até o M6. Em resumo, a incidência de tremor foi maior nos do grupo SA.

Com relação à PAS, não houve diferença significativa $(\mathrm{P}>0,05)$ entre grupos e entre momento de avaliação (Tab. 1). A variação para menos da PAS no M30 nos animais dos grupos FE e SA ocorreu em virtude de quatro animais $(66,7 \%)$ de cada grupo apresentarem valores de PAS abaixo dos valores da normalidade para a espécie. No grupo SA em M2 e M6 três animais (50\%) e no grupo FE em M2 e dois em M6 permaneceram com valores de PAS abaixo dos valores de referência.

Zanoni e Palhares (2002) relataram diminuição da pressão arterial em crianças na primeira hora após uso do fenoterol com aumento progressivo no final da segunda hora, resultado semelhante, em parte, com os do presente estudo, em que foi observada diminuição da PAS 30 minutos após o uso dos fármacos, grupos FE e SA; no entanto, essa diminuição permaneceu até M6 somente no grupo SA.

Com relação à FR, foi observado aumento dos valores no M30 nos animais dos grupos FE $(33,3 \%)$ e SA (50\%) (Tab. 1). Observou-se, também, que dos três cães do grupo SA, um apresentou elevação da FR em três momentos, inclusive no $\mathrm{MC}$, e no grupo $\mathrm{FE}$ isto ocorreu em todos os momentos, e também no MC. O aumento da FR no animal do FE em um dos animais do SA em MC pode ter sido em decorrência do estresse de contenção e manipulação para aferição dos parâmetros, por serem animais um pouco mais agitados que os demais. Entretanto, a frequência neles aumentou ainda mais no M30 e permaneceu acima dos valores normais para espécie até M6 e M2 nos grupos FE e SA, respectivamente. Para o grupo FE, outros dois apresentaram aumento da FR em M30 até M2 e M2 até M6. Para o grupo SA, um segundo animal apresentou aumento da FR em M30, que se manteve elevada até M6.

Observou-se que a média da temperatura retal não se alterou nos animais de ambos os grupos em nenhum momento de avaliação (Tab. 1). Para dois animais $(33,3 \%)$ do grupo FE, observou-se pequena elevação da temperatura em M30, e assim permaneceu até M6.

$\mathrm{Na}$ avaliação da hemogasometria, os valores encontrados permaneceram dentro da normalidade para a espécie, e não foi observada diferença significativa $(\mathrm{P}>0,05)$ entre grupos e momentos (Tab. 2). Estudo realizado por Burggraaf et al. (2001) relatou alterações na saturação de oxigênio após inalação com o salbutamol em pacientes humanos asmáticos.

Nos grupos FE e SA, em todos os animais estudados, para duração da onda $\mathrm{P}(\mathrm{mV})$, duração do intervalo PR (s), duração do complexo QRS (s) e intervalo QT (s), não houve diferença significativa $(\mathrm{P}>0,05)$ entre grupos $\mathrm{e}$ momentos. O ritmo cardíaco caracterizou-se por ritmo sinusal normal e apenas um animal do grupo SA apresentou bloqueio sinusal M2.

Alguns autores relataram em humanos que receberam salbutamol, tanto pela via intravenosa 
como inalatória, prolongamento do intervalo QT secundário à hipocalemia e taquiarritmias (Santana et al., 2001; Abramson et al., 2003). Autores relataram em pacientes com obstrução das vias aéreas inferiores maior tendência de o fenoterol, em relação ao salbutamol, induzir ao aparecimento de arritmias ventriculares e ao efeito cronotrópico, originando alterações eletrofisiológicas, como o aumento do intervalo QT (Vieira et al., 2000).
As duas vias de administração mostraram-se eficientes, já que foram observados todos os efeitos secundários à administração dos dois fármacos. No entanto, na comparação das duas técnicas utilizadas, foi observada maior facilidade de administração, menor custo e menor tempo de administração do salbutamol por meio do inalador dosimetrado, do que do fenoterol, por meio do nebulizador, sendo este resultado semelhante ao observado por Amantéa et al. (2002).

Tabela 1. Média e desvio-padrão da frequência cardíaca (FC), frequência respiratória (FR), temperatura retal (TR) e pressão arterial sistólica (PAS) dos cães dos grupos FE (fenoterol) e SA (salbutamol) nos diferentes momentos $(\mathrm{M})$ de avaliação

\begin{tabular}{cccccc} 
Variável & Grupo & $\begin{array}{c}\text { MC } \\
\text { (controle) }\end{array}$ & $\begin{array}{c}\text { M30 } \\
\text { (após 30min) }\end{array}$ & $\begin{array}{c}\text { M2 } \\
\text { (após 2h) }\end{array}$ & $\begin{array}{c}\text { M6 } \\
\text { (após 6h) }\end{array}$ \\
\hline FC & FE & $138 \pm 27,77$ & $145,00 \pm 21,68$ & $136,67 \pm 14,08$ & $122,05 \pm 14,05$ \\
$($ bpm) & SA & $130 \pm 11,40 A$ & $152,00 \pm 29,93$ & $158,33 \pm 33,71 \mathrm{~B}$ & $138,33 \pm 22,95$ \\
FR & FE & $36,67 \pm 5,89$ & $37,33 \pm 17,47$ & $34,67 \pm 12,56$ & $30,67 \pm 6,89$ \\
$($ mpm) & SA & $33,67 \pm 14,50$ & $41,00 \pm 21,16$ & $35,33 \pm 8,55$ & $32,67 \pm 8,16$ \\
TR & FE & $39,00 \pm 0,30$ & $39,15 \pm 0,66$ & $38,09 \pm 0,57$ & $38,77 \pm 0,58$ \\
$\left({ }^{\circ} \mathrm{C}\right)$ & SA & $38,82 \pm 0,26$ & $38,57 \pm 0,47$ & $38,45 \pm 0,12$ & $38,33 \pm 0,10$ \\
PAS & FE & $115,00 \pm 13,78$ & $96,67 \pm 17,51$ & $111,67 \pm 17,22$ & $113,33 \pm 13,66$ \\
$(\mathrm{mmHg})$ & SA & $118,33 \pm 10,67$ & $93,33 \pm 18,62$ & $100 \pm 16,73$ & $95,00 \pm 13,78$ \\
\hline
\end{tabular}

Letras maiúsculas diferentes na linha indicam diferença entre momentos, dentro do mesmo grupo.

Tabela 2. Média e desvio-padrão da hemogasometria dos cães dos grupos FE (fenoterol) e SA (salbutamol) nos diferentes momentos (M) de avaliação

\begin{tabular}{llcccc}
\hline Variável & Grupo & $\begin{array}{c}\text { MC } \\
\text { (controle) }\end{array}$ & $\begin{array}{c}\text { M30 } \\
\text { (após 30min) }\end{array}$ & $\begin{array}{c}\text { M2 } \\
\text { (após 2h) }\end{array}$ & $\begin{array}{c}\text { M6 } \\
\text { (após 6h) }\end{array}$ \\
\hline $\mathrm{pH}(\mathrm{U})$ & FE & $7,36 \pm 0,04$ & $7,35 \pm 0,06$ & $7,33 \pm 0,05$ & $7,37 \pm 0,04$ \\
& SA & $7,38 \pm 0,03$ & $7,37 \pm 0,02$ & $7,39 \pm 0,05$ & $7,38 \pm 0,05$ \\
$\mathrm{PCO}_{2}(\mathrm{~mm} / \mathrm{Hg})$ & FE & $41,65 \pm 2,50$ & $43,23 \pm 7,23$ & $40,76 \pm 3,64$ & $42,76 \pm 5,32$ \\
& SA & $41,76 \pm 4,18$ & $40,75 \pm 2,71$ & $39,05 \pm 1,67$ & $42,73 \pm 5,70$ \\
$\mathrm{PO}_{2}(\mathrm{~mm} / \mathrm{Hg})$ & FE & $37,00 \pm 5,72$ & $42,56 \pm 1,75$ & $44,25 \pm 9,88$ & $40,85 \pm 8,02$ \\
$\mathrm{HCO}_{3}$ & SA & $38,01 \pm 4,05$ & $39,38 \pm 2,13$ & $40,85 \pm 4,52$ & $48,33 \pm 10,94$ \\
$(\mathrm{mEq} / \mathrm{L})$ & FE & $23,48 \pm 1,16$ & $23,28 \pm 2,57$ & $22,11 \pm 2,01$ & $24,18 \pm 3,24$ \\
\hline
\end{tabular}

Valores de referência: $\mathrm{pH}(\mathrm{U})=7,33-7,45 ; \mathrm{PCO}_{2}(\mathrm{~mm} / \mathrm{Hg})=36-48 ; \mathrm{PO}_{2}(\mathrm{~mm} / \mathrm{Hg})=40-50 ; \mathrm{HCO}_{3}=17-25$

\section{CONCLUSÕES}

O fenoterol provocou menor estimulação cardíaca e tremores comparados ao salbutamol, foi mais seguro e houve maior facilidade, menor custo e menor gasto de tempo na administração do salbutamol por meio do inalador dosimetrado em relação ao fenoterol por nebulização.

\section{REFERÊNCIAS}

ABRAMSON, M.J.; WALTERS, J.; WALTERS, E.H. Adverse effects of beta-agonists: are they clinically relevant? Am. J. Resp. Med., v.2, p.287-297, 2003.

AMANTÉA, S.L.; SANCHEZ, I.; PIVA, J.P. et al. Controvérsias no manejo farmacológico da asma aguda infantil. J. Pediatr., v.78, p.151-160, 2002. 
BURGGRAAF, J.; WESTWNDORP, R.G.J.; VEEN, J.C.C.M. et al. Cardiovascular side effects of inhaled salbutamol in hypoxic asthmatic patients. Thorax, v.56, p.567-569, 2001.

GONZÁLES-MUNOZ, C.; FUENTE, T.; HERNANDEZ-CASCALES, J. Phosphodiesterase inhibition unmask a positive inotropic effect mediated by beta 2 -adenoceptors in rat ventricular myocardium. Eur. J. Pharmacol., v.607, p.151-155, 2009.

MENDES-CAMPOS, L.E.; BIAGIONI, C.M. Efeito broncodilatador do salbutamol inalado através de espaçadores com e sem tratamento antiestático. J. Pneumol., v.27, p.249-254, 2001.

PAGANO, M.; GAUVREAU, K. Princípios de bioestatística. Rio de Janiero: Livros Técnicos e Científicos, 2004. 136p.

ROTTA, E.T.; AMANTÉA, S.L.; FROEHLICH, P.E. et al. Determinação das concentrações plasmáticas de salbutamol pós-nebulização em serviço de emergência pediátrica. J. Pediatr., v.83, p.481-484, 2007.

SANTANA, J.C.; BARRETO, S.S.M.; PIVA, J.P. et al. Estudo controlado do uso de salbutamol no tratamento precoce da crise de asma aguda grave em crianças. J. Pediatr., v.77, p.123-126, 2001.
SILVA, M.B.; DUVIVIER, D.H.; VOTION, D. et al. Aerossolterapia nas doenças respiratórias em equinos. Braz. J. Vet. Res. Anim. Sci., v.38, p.88-96, 2001.

SOUZA, H.J.M.; ROSSI, C.N. Emergências Respiratórias em gatos. In: RABELO, R.C.; CROWE, D.T. Fundamentos de terapia intensiva veterinária em pequenos animais: condutas do paciente crítico. Rio de Janeiro: LF Livros, 2005. p.411-437.

TSOI, A.N.; APKHIPOV, W. Pharmacodynamics of inhalation broncholytic agents introduced in a single dose by nebulizer in patients with severe exacerbation of bronchial asthma. Ter. Arkh., v.74, p.17-21, 2002.

VIEIRA, S.E.; GILIO, A.E.; LOTUFO, J.P.B. et al. Há potencial letal decorrente da inalação de fenoterol pela criança asmática? Pediatria, v.22, p.286-294, 2000.

ZANGIROLAMI FILHO, D.Z; DE SOUZA, W.A.; MEIRA, F.Q. et al. Doença pulmonar obstrutiva crônica. Rev. Cient. Eletr. Med. Vet., v.10, p.10-17, 2008.

ZANONI, L.Z.; PALHARES, D.B. Inalação contínua com fenoterol na criança com asma aguda grave: efeitos clínicos imediatos. J. Pediatr., v.78, p.423-428, 2002. 